\title{
Central nervous system blastomycosis presenting as a year-long chronic headache
}

\author{
Elena Grebenciucova ${ }^{1}$, Maciej S. Lesniak ${ }^{2}$, Peter Pytel ${ }^{3}$, Rimas V. Lukas ${ }^{1}$ \\ ${ }^{1}$ Department of Neurology, University of Chicago Medical Center, Chicago, IL 60637, USA. \\ ${ }^{2}$ Department of Neurosurgery, Northwestern University, Chicago, IL 60637, USA. \\ ${ }^{3}$ Department of Pathology, University of Chicago Medical Center, Chicago, IL 60637, USA.
}

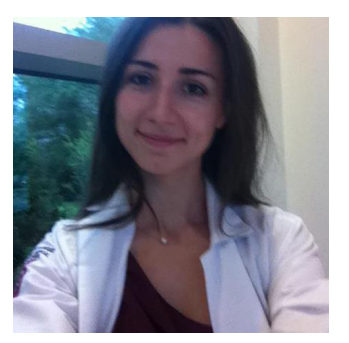

Dr. Elena Grebenciucova is currently a senior neurology resident at the University of Chicago Medical Center. Her interests lie in the field of neuroimmunology and neuro-infectious disorders. This year, she will continue serving patients with neuroinflammatory diseases in a new capacity, as a Neuroimmunology fellow at the University of Pennsylvania.

\section{A B S T R A C T}

This case describes a posterior fossa mass due to blastomycotic infection in a non-immunocompromised 41-year-old male presenting with a chronic headache for over one year. Given the risk of herniation, no lumbar puncture could be performed. A full work-up found no evidence of systemic infection. Surgical resection helped identify the mass as a blastomycotic abscess. Magnetic resonance imaging characteristics of the mass were helpful in the identification of the mass as a fungal abscess.

Key words: CNS blastomycosis; blastomycotic abscess; chronic headache; posterior fossa mass; fungal abscess

\section{INTRODUCTION}

Blastomycosis isolated to the central nervous system(CNS) is exceedingly rare. In the absence of other more common systems' involvement (such as lungs), blastomycosis is rarely considered high on the differential. This case describes a nonspecific presentation of a blastomycotic abscess as a chronic headache and discusses the difficulties associated with the prompt diagnosis of this disease. The scope of this case is to demonstrate that when presenting as a posterior fossa mass, blastomycotic abscesses have a vast differential and ultimately require a biopsy or resection for the correct diagnosis. MRI imaging may be helpful in helping distinguish fungal abscesses from their neoplastic or bacterial counterparts.

Corresponding Author: Dr. Elena Grebenciucova, Department of Neurology, University of Chicago Medical Center, Chicago, IL 60637, USA. Email: elena.grebenciucova@uchospitals.edu

\begin{tabular}{|l|l|}
\hline \multicolumn{2}{|c|}{ Access this article online } \\
\hline Quick Response Code: & Website: \\
\hline & www.nnjournal.net \\
\cline { 2 - 3 } & Dol: 10.20517/2347-8659.2015.26 \\
\hline
\end{tabular}

\section{CASE REPORT}

A 41 years old male from rural Illinois with a history of hypertension and alcohol abuse presented for evaluation of 16 months of progressive dull occipital headaches, unresponsive to pain medications. Over the prior 6 months the patient developed nausea with rapid head movements as well as dysarthria and right hand clumsiness. Non-contrast computed tomography (CT) of the brain demonstrated an ill-defined mixed density lesion in the cerebellum [Figure 1]. Magnetic resonance imaging (MRI) revealed a $3.5 \mathrm{~cm} \times 2.6 \mathrm{~cm}$ intra-axial mass in the midline superior cerebellum abutting the tentorium and compressing the 4 th ventricle. The mass was mildly hyperintense on T1-weighted images and heterogeneous but predominately hypointense on $\mathrm{T} 2 /$ FLAIR [Figure 2]. It demonstrated significant internal susceptibility on susceptibility weighted images compatible with vascularity and/or hemorrhage. There

This is an open access article distributed under the terms of the Creative Commons Attribution-NonCommercial-ShareAlike 3.0 License, which allows others to remix, tweak, and build upon the work non-commercially, as long as the author is credited and the new creations are licensed under the identical terms.

For reprints contact: service@oaepublish.com

Cite this article as: Grebenciucova E, Lesniak MS, Pytel P, Lukas RV. Central nervous system blastomycosis presenting as a year-long chronic headache. Neuroimmunol Neuroinflammation 2016;3:17-9.

Received: 12-06-2015; Accepted: 01-10-2015 


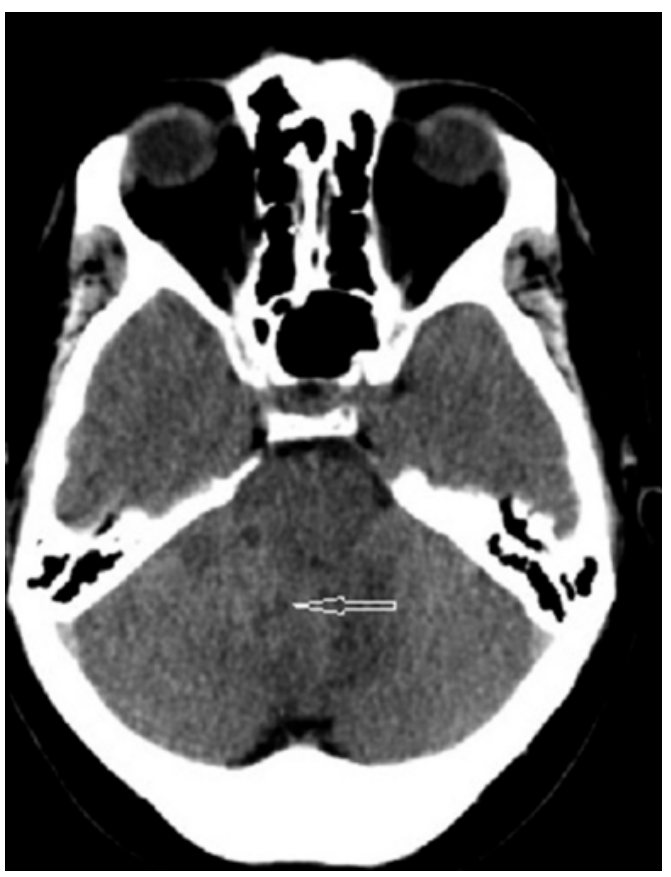

Figure 1: Axial head computed tomography image revealing a predominantly hypodense mass lesion in the cerebellum, centered at the vermis. The lesion compresses the fourth ventricle (arrow pointing to the mass)

was avid internal enhancement of the lesion on postcontrast images. Additionally there was a thin tract of contrast enhancement extending from the lesion along the right lateral margin of the brainstem. There was mild edema of the surrounding cerebellum.

Differential diagnosis of the posterior fossa mass in a 41 years old male included neoplastic (high- or lowgrade gliomas, medulloblastoma, hemangioblastoma, lymphoma and infectious etiologies. Pilocytic astrocytomas (WHO grade I) and medulloblastomas (WHO grade IV) occur more frequently in the pediatric population. CNS lymphomas occur more frequently in the elderly or HIV+ population. Hemangioblastomas often occur in association with Von Hippel-Lindau syndrome. Other than the suspicion of alcohol abuse, patient was otherwise not immunocompromised. He was HIV negative, lacked peripheral leukocytosis, fevers, and had an unremarkable CT of the chest. Given the location, the size, and the mass effect of the lesion, no lumbar puncture could be pursued due to the risk of herniation. The patient underwent a suboccipital craniotomy achieving a complete resection. Pathology showed granulomas with fungal organisms. Periodic acid-Schieff and Giemsa (GMS) stains revealed rounded yeast forms consistent with Blastomycosis dermatitidis [Figure 3]. Post-operative cerebrospinal fluid (CSF) revealed $>1,000$ White blood cells with neutrophilic predominance, normal glucose at 64 and high protein at 300 .

\section{DISCUSSION}

Blastomycosis is a pyogranulomatous infection that
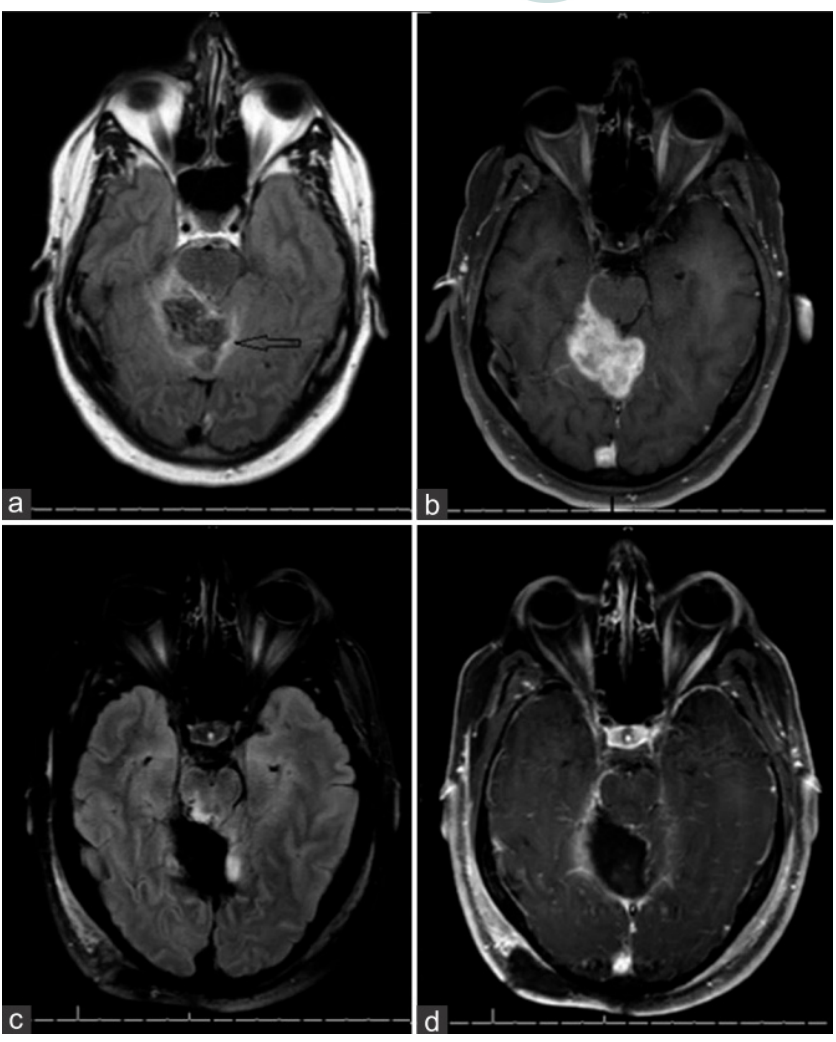

Figure 2: Axial magnetic resonance imaging images of fluid-attenuated inversion recovery (a) and T1 postcontrast (b) at initial presentation and then postoperatively ( $c$ and $\mathrm{d}$ ) revealing complete radiographic resection of the lesion

occurs predominantly by inhalation of the microconidia of the dimorphic fungus $B$. dermatitidis. The fungus is prevalent in the areas along the Mississipi and Ohio river basins as well as Midwestern regions bordering the Great Lakes. About 91\% of infections are pulmonary with subsequent dissemination to the skin, bone or genitourinary system. CNS involvement occurs only rarely $5-10 \%$ of cases. ${ }^{[1]}$ Isolated CNS blastomycosis is rare. Infection occurs in both immunocompetent and immunosuppressed hosts. For example, in a case series of 22 patients with CNS blastomycosis, only 12 patients were immunocompromised (i.e. HIV, chronic steroid use, antitumor necrosis factor therapy for more than 6 months). ${ }^{[2]}$ Clinical and experimental evidence (predominantly animal studies) suggests that chronic alcohol consumption significantly alters many lines of immune system and predisposes alcoholics to an increased risk of infection, increased morbidity, and mortality. ${ }^{[3]}$

CNS blastomycotic infection can present as either acute or indolent meningoencephalitis. At times, the only symptoms are intractable headaches. In a recent study that evaluated outcomes of 16 patients with CNS blastomycosis, the most frequent symptoms at presentation were headaches or a focal neurologic deficit in 63 and $56 \%$ of patients respectively. ${ }^{[4]}$ Other manifestations include leptomeningeal involvement, single or multiple abscesses intracranially or in the spinal cord, as well as in the epidural space causing cord compression. On the MRI these may present as a single or multiple lesions 

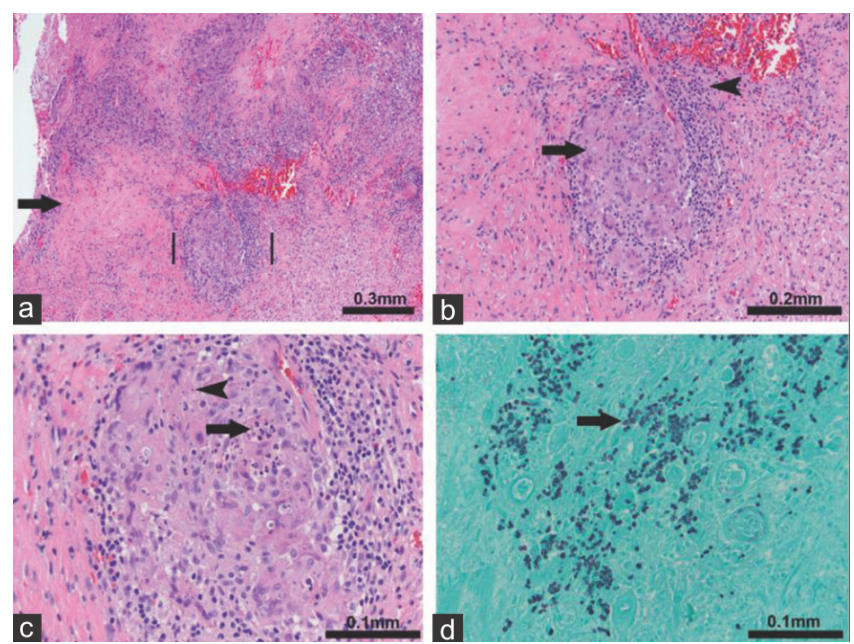

Figure 3: Histologic analysis of the biopsy sample. (a) Histologic sections show a background of reactive fibrosis (arrow) with nodular inflammatory cell infiltrates (between vertical lines). Higher magnification ( $\times 10)$; (b) chronic inflammatory cells: small mononuclear cells (arrow head) and nodular aggregates of pale pink "epithelioid" histiocytes (arrow) imparting a granulomatous appearance $(\times 20)$; (c) Small spherical organisms are seen (arrow head) and focally a few neutrophils are present in the center of the granuloma (arrow, $\times 40$ ); (d) GMS staining confirms the presence of fungal organisms that morphologically appear as small dark stained round yeast forms (arrow). Blastomyces dermatitidis $(\times 40)$

producing mass effect (particularly in the cerebellum), diffuse leptomeningeal enhancement, cerebritis or obstructive hydrocephalus. Restricted diffusion is frequently one of the earliest MRI findings with fungal abscesses. This occurs due to an increased cellularity and viscosity of the pus associated with the infection and frequently precedes gadolinium enhancement. Reduced diffusion signal may frequently be heterogeneous. In smaller lesions, it may be punctate. When compared with fungal infections, bacterial abscesses tend to have a highly restricting homogeneous center. In contrast to their marked diffusion abnormality, fungal abscesses may demonstrate only a weak ring enhancement. This is thought to be secondary to a weak peripheral immune response. A combination of ring enhancement and diffusion signal can help differentiate fungal abscesses from bacterial abscesses or brain metastases. Brain metastases tend to have a thicker ring enhancement and a reduced diffusion in the necrotic center. Brain metastases frequently have a thicker ring enhancement, but typically have no reduced diffusion in the necrotic center. ${ }^{[5]}$

Definitive diagnosis is established either by isolation of the fungus from a culture or direct visualization on the histological slides. Isolation from the CSF is uncommon. In a case series of 22 patients with CNS blastomycosis, CSF cultures were positive only in 2 patients. ${ }^{[2]}$ Serologic testing is generally considered not to be useful in blastomycosis due to high cross-reactivity with other endemic mycoses. Antigen testing may be positive in the urine and serum. PCR is rarely used and typically not commercially available.

Thus, most cases require a biopsy and a histopathologic examination of the tissue to arrive at the correct diagnosis. The case described above had negative serology, CSF culture and required a tissue sample obtained during resection to diagnose it as a blastomycosis abscess.

Financial support and sponsorship

Nil.

\section{Conflicts of interest}

There are no conflicts of interest.

\section{REFERENCES}

1. Chapman W, Lin AC, Hendricks KA, Nolan RL, Currier MM, Morris KR, Turner HR. Endemic blastomycosis in Mississippi: epidemiological and clinical studies. Semin Respir Infect 1997;12:219-28.

2. Kravitz GR, Davies SF, Eckman MR, Sarosi GA. Chronic blastomycotic meningitis. Am J Med 1981;71:501-5.

3. Szabo G, Mandrekar P. A recent perspective on alcohol, immunity, and host defense. Alcohol Clin Exp Res 2009;33:220-32.

4. Bush JW, Wuerz T, Embil JM, Del Bigio MR, McDonald PJ, Krawitz S. Outcomes of persons with blastomycosis involving the central nervous system. Diagn Microbiol Infect Dis 2013;76:175-81.

5. Starkey J, Moritani T, Kirby P. MRI of CNS fungal infections: review of aspergillosis to histoplasmosis and everything in between. Clin Neuroradiol 2014;24:217-30. 